\title{
Global Health
}

\section{Pediatric spinal cord injury rehabilitation: A protocol for an international multicenter project (SINpedSCI)}

Kirsti Skavberg Roaldsen ${ }^{\mathrm{a}, \mathrm{b}, \mathrm{c}, *}$, Vivien Jørgensen ${ }^{\mathrm{a}}$, Wiebke Höfers ${ }^{\mathrm{a}, \mathrm{b}}$, Susanne Sällström ${ }^{\mathrm{a}}$, Marika Augutis $^{\mathrm{d}, \mathrm{e}}$, Per Ertzgaard ${ }^{\mathrm{f}, \mathrm{g}}$, Kerstin Wahman ${ }^{\mathrm{d}, \mathrm{e}}$, Mona Strøm ${ }^{\mathrm{h}}$, Kristine Marie Vege ${ }^{\mathrm{h}, \mathrm{v}}$, Kristine Sørland ${ }^{\mathrm{h}}$, GenLin Liu ${ }^{\mathrm{i}}$, Qi Zhang ${ }^{\mathrm{j}}$, Yu-Xi Yang ${ }^{\mathrm{k}}$, Yang Chen ${ }^{\mathrm{k}}$, Olga Zakharova ${ }^{\mathrm{l}}$, Zinaida Trukhankina ${ }^{\mathrm{m}}$, Atheer Ghatasha ${ }^{\mathrm{n}}$, Eman Hamdan ${ }^{\mathrm{n}}$, Tal Krasovsky,p, Dafna Guttman ${ }^{\mathrm{o}}$, Katharina Stibrant Sunnerhagen ${ }^{\mathrm{q}}$, Peter W. New ${ }^{\mathrm{r}, \mathrm{s}, \mathrm{t}}$, Tamara Bushnik ${ }^{\mathrm{u}}$, Renat Sukhov ${ }^{\mathrm{u}}$ and Johan K. Stanghelle ${ }^{\mathrm{a}, \mathrm{v}}$

${ }^{a}$ Department of Research, Sunnaas Rehabilitation Hospital, Nesodden, Norway

${ }^{\mathrm{b}}$ Department of Physiotherapy, Faculty of Health Sciences, Oslo Metropolitan University, Oslo, Norway

${ }^{\mathrm{c}}$ Department of Neurobiology, Care Sciences and Society, Division of Physiotherapy, Karolinska Institute, Stockholm, Sweden

${ }^{\mathrm{d}}$ Department of Neurobiology, Care Sciences and Society, Division of Neurogeriatrics, Karolinska Institute, Stockholm, Sweden

${ }^{\mathrm{e}}$ Research and Development Unit, Rehab Station Stockholm, Stockholm, Sweden

${ }^{\mathrm{f}}$ Department of Rehabilitation Medicine and Department of Medical and Health Sciences, University Hospital, Linköping, Sweden

${ }^{\mathrm{g}}$ H.R.H. Crown Princess Victoria's Children and Youth Hospital, Linköping Hospital, Sweden

${ }^{\mathrm{h}}$ Department for Spinal Cord Injury, Unit for Children and Adolescents, Sunnaas Rehabilitation Hospital, Nesodden, Norway

${ }^{\mathrm{i}}$ Department of Spinal Cord Injury Rehabilitation, China Rehabilitation Research Center (CRRC) and Capital Medical University School of Rehabilitation Medicine, Beijing Bo'ai Hospital, Beijing, China

${ }^{\mathrm{j}}$ Department of Physical Therapy, China Rehabilitation Research Center (CRRC) and Capital Medical University School of Rehabilitation Medicine, Beijing Bo'ai Hospital, Beijing, PR China

${ }^{\mathrm{k}}$ SCI Rehabilitation, Bayi Rehabilitation Center, Yongning Town, Chengdu, PR China

${ }^{1}$ Children's City Hospital, Petrozavodsk, Karelia, Russia

${ }^{\mathrm{m}}$ Republican Children's Hospital, Petrozavodsk, Karelia, Russia

${ }^{\mathrm{n}}$ Bethlehem Arab Society for Specialized Rehabilitation and Surgery, Bethlehem, Palestine

${ }^{\circ}$ Department of Pediatric Rehabilitation, The Edmond \& Lily Safra Children's Hospital, Chaim Sheba Medical Center (SMC), Tel-Hashomer, Israel

${ }^{\mathrm{p}}$ Department of Physical Therapy, University of Haifa, Haifa, Israel

${ }^{\mathrm{q}}$ Department of Clinical Neuroscience at Institute of Neuroscience and Physiology, Sahlgrenska Academy at University of Gothenburg, Gothenburg, Sweden

\footnotetext{
${ }^{*}$ Corresponding author: Kirsti Skavberg Roaldsen, Karolinska Institutet, Department of Neurobiology, Care Sciences and Society, Division of Physiotherapy. Address: Box 23400, SE-141 83 Huddinge, Sweden. Second address: Sunnaas Rehabilitation
}

Hospital, Department of Research. Bjørnemyrveien 11, NO-1453 Bjørnemyr, Norway. Tel.: +46 708776790 and +47 92083476, E-mail: kirsti.skavberg.roaldsen@ki.se and kirroa@sunnaas.no. 
${ }^{\mathrm{r}}$ Department of Rehabilitation, Spinal Rehabilitation Service, Caulfield Hospital, Alfred Health, Melbourne, Australia

${ }^{\mathrm{s}}$ Epworth-Monash Rehabilitation Medicine Unit, Monash University, Melbourne, Victoria, Australia

${ }^{\mathrm{t}}$ Department of Epidemiology and Preventive Medicine, School of Public Health \& Preventive Medicine, Monash University, Victoria, Australia

" Rusk Rehabilitation, New York University Langone Health, New York, NY, USA

${ }^{\mathrm{v}}$ Institute of Clinical Medicine, Faculty of Medicine, University of Oslo, Oslo, Norway

Received 12 November 2020

Accepted 19 October 2021

\begin{abstract}
.
PURPOSE: Children and adolescents ( $<18$ years old) who sustain a spinal cord injury (SCI) should ideally be managed in specialized rehabilitation services. This project aims to describe the organization of pediatric SCI in ten rehabilitation units in seven countries and to qualitatively explore psychosocial aspects of adolescents living with SCI.

METHODS: A multicenter cross-sectional project is planned, using quantitative (web survey) and qualitative (interview) methods in ten rehabilitation units from Norway, Sweden, United States, Israel, PR China, Russia and Palestine. Individual interviews will be conducted with $\geq 20$ adolescents aged 13-17 years at least 6 months' post rehabilitation.

RESULTS: Units involved will be described and compared, according to funding, attachment to an acute SCI unit, catchment area, number of beds, admittance and discharge procedures, availability of services, staff/patient ratio, content and intensity of rehabilitation programs, length of stay, measurement methods, follow-up services, health promotion services, and pediatric SCI prevention acts. The semi-structured interview guide will include experiences from acute care and primary rehabilitation, daily life, school, contact with friends, leisure time activities, peers, physical and psychological health, and the adolescents' plans for the future.
\end{abstract}

CONCLUSION: Based on the present protocol, this project is likely to provide new insight and knowledge on pediatric SCI rehabilitation and increase the understanding of pediatric SCI in adolescents and their families internationally.

Keywords: Children, adolescents, spinal cord injury, multidisciplinary, interdisciplinary, specialized rehabilitation, management, international, multicenter, patient perspective, psychosocial aspects

\section{The significance and applicability of this protocol article}

Collaboration across borders is paramount to capture multicultural similarities as well as differences within pediatric spinal cord injury rehabilitation. A protocol article like this is important to create awareness of ongoing international multicenter research within the field.

To achieve the best possible collaboration across borders and have the possibility to solve misunderstandings as early as possible, it is vital to develop a friendly and open atmosphere of trust and to choose a simple design based on level of expertise. It is important to discuss procedures and project-specific documents together with research partners to reach consensus and to develop a common platform of understanding. A physical workshop should start the collaboration and be followed up by regular digital contacts in order to facilitate collaboration, especially in relation to non-traditional partners. Financial agreement between research part- ners should be signed prior to project start. Author lists needs to be discussed in advance and be in accordance with Vancouver recommendations.

\section{Background}

Spinal cord injury (SCI) in the pediatric population $(<18$ years old) is relatively rare but has significant physiological and psychological consequences that have a life-long impact on most aspects of daily life. The injury can be traumatic or non-traumatic, caused by infection, inflammation, embolism, tumors, or bleeding. Most traumatic injuries are from preventable causes such as traffic accidents, falls, sports/recreation accidents, and violence. The mechanism of injury, the male/female ratio, and the level of injury differs from the adult SCI population [1]. The rate of recovery is also considered to be faster in the pediatric population compared with other age groups [2]. 
There is a scarcity of population-based epidemiological data on the incidence of pediatric SCI, both traumatic and especially non-traumatic. For traumatic pediatric SCI, the incidence rates vary widely across the world. The risk of sustaining a traumatic SCI is lowest for children younger than 16 years and increases with age [3]. According to a literature review by New et al. [4], the median incidence rates of traumatic SCI (cases/million population/year) for the pediatric population (0-15 years old) in global regions are as follows: Western Europe, 3.3; Asia, 5.4; Australia, 9.9; North America, 13.2. Worldwide, the most frequent causes are traffic accidents (46-74\%), falls (12-35\%), and sport/recreation (10-25\%). Regarding non-traumatic injuries, the median incidence rate is 6.2 in Western Europe and 2.1 in North America, mostly caused by tumors (30-63\%) and inflammatory/autoimmune causes (28-35\%) [4].

A SCI is typically a devastating event, leading to major loss of functions and commonly giving rise to complex and life-long physical disability with loss of sensitivity, disturbed functions in many body systems, and increased vulnerability to medical and psychological complications. Thus, an SCI has a lifechanging impact on the injured person and the family, leading to hurdles for life fulfillment. Individuals with a pediatric onset of SCI have comprehensive and highly complex rehabilitation needs because the injury coincides with the ongoing physical, social, and emotional development of the young person [5, 6]. Consequently, all young persons with SCI should ideally be managed according to specialized highcost/low-volume rehabilitation services that provide highly complex rehabilitation after illness or injury that is beyond the scope of local general and specialist services [7-9]. There is no internationally accepted definition of specialized SCI rehabilitation. An operational definition for all ages has been provided by Spinal Cord Injury Research Evidence (SCIRE): "A specialized SCI rehabilitation program provides comprehensive, individualized, and patient focused rehabilitation services, for inpatient, transitional living, outpatient and follow-up care, to empower people with SCI and their families to achieve optimal quality of life continuing into the community (focusing on increasing self-reliance and gaining independence)" [10].

Specialized pediatric SCI rehabilitation services require a high level of medical expertise in relevant areas. The service includes both assessment, treatment, guidance, and training delivered by a multi-professional and interdisciplinary team who have undergone recognized specialist training in pediatric as well as SCI rehabilitation. Specialized rehabilitation is normally provided in coordinated service networks planned over a regional population of 1-3 million through collaborative (specialized) commissioning arrangements [9].

There is strong research-based evidence to show that rehabilitation in specialized settings for people with SCI is effective and provides value in terms of reducing length of stay in the hospital and reducing the costs of long-term care, as well as reducing the risk for long-term complications [11-14]. Sufficient scope and intensity of the rehabilitation services are crucial from the initial care throughout the whole rehabilitation course. Also, specialized pediatric SCI rehabilitation is in accordance with the World Health Organization's (WHO's) rules and regulations for children in hospital, which ensures the basic rights of children and adolescents in health care in line with the Convention on the Rights of the Child $[15,16]$. Three basic rules for the management of pediatric SCI have been proposed to optimize rehabilitation: (1) treat the whole family; (2) use a multidisciplinary approach; and (3) view treatment as an ongoing dynamic process that must change as the child grows and develops [1]. The American Spinal Injury Association (ASIA) has published guidelines for good pediatric SCI rehabilitation, which aim to guide parents to choose the best options for their child [17].

Due to the low incidence, children and adolescents with SCI are widely dispersed, resulting in large regional and national differences in management. Hence, many children and adolescents with SCI may experience disparity of care. This is concerning because young people live longer with SCI than people with adult onset and are therefore more susceptible to long-term complications [3, 18, 19]. Follow-up rehabilitation has to be a life-long process, flexible, and adapted to functional improvements, complications and changing demands. Nevertheless, juvenile age appears to be an independent predictor for better functional outcome after SCI [20].

To better meet the specific needs and demands of persons in this age group, the young person's own experiences of living with SCI must be explored to inform health care and research on agenda-setting activities [21]. Involvement of the individual in decisions concerning their own life is therefore important within rehabilitation. Unfortunately, feedback from patients and their organizations shows that patient participation is still deficient [8]. Adolescents aged 
10-17 years are especially vulnerable because they are experiencing major biological, psychological and social changes in the transition from childhood to adulthood [22]. Thus, psychosocial issues are critical factors in successful adjustment to a life with SCI and place great demands on the organization of care and management for pediatric SCI worldwide [6, 22-24].

It has been suggested that non-clinical factors, such as hospital processes and systems of care as well as the availability and timing of resources and services, influence the outcomes of rehabilitation beyond clinical factors such as age at the time of injury and injury severity [25]. Therefore, description of the health care setting is an important component of research and is included in the STROBE Statement, which provides guidelines for authors on reporting observational studies and recommends describing the setting, location, participant details, and source and method of selection of patients [26]. Moreover, according to New et al. [25], the non-clinical factors that should be reported in specialized SCI rehabilitation studies include referral patterns, selection criteria for admission [27], the range of therapy options, the intensity of therapy [28], and post primary rehabilitation follow-up services including programs for health maintenance and prevention. Comparing studies conducted in different settings that do not describe the setting in adequate detail therefore has limited value. Only one international comparative study of the services and types of rehabilitation programs available for pediatric SCI has been identified [29].

A comprehensive model of care for specialized pediatric SCI rehabilitation does not exist. Also, access to rehabilitation services is unevenly distributed between countries and continents [30]. The content of rehabilitation may therefore vary significantly internationally because nations and regions differ in geography, population density, and economic resources, resulting in inequality of rehabilitation services for children and adolescents. In addition, there has been a lack of agreed quality indicators for pediatric SCI care. Since 2006, work with the International SCI Basic Data Sets has been ongoing to provide standardization in recording variables that are significant for SCI care [30, 31]. Several of these data sets have been reviewed to identify questions relevant for the pediatric population. In addition, new data sets have been designed specifically for the pediatric population [32-34], which has improved the ability to compare and combine data. However, the content of specialized pediatric SCI rehabilitation has not been standardized or described; only one study [29] has tried to compare the care and rehabilitation services for pediatric SCI in European countries. There are reasons to believe that physical, social, financial, organizational, and attitudinal diversities may influence how specialized pediatric SCI rehabilitation services are provided [35]. Therefore, a study comparing specialized pediatric SCI rehabilitation in international institutions, both from the perspective of the health care providers and the individual adolescent receiving rehabilitation, has the potential to provide new knowledge. The present project protocol aims to gain insight in delivery of pediatric SCI rehabilitation as well as prerequisites for services and optimal specialized pediatric rehabilitation. How these services are organized, provided, and perceived by adolescents with SCI will be illuminated. Requirements for referral and discharge, ideal staff/patient ratios, evaluation of rehabilitation outcomes and follow-up services are not yet standardized and thus need to be described and discussed.

To broaden understanding of pediatric SCI rehabilitation models of care, more information and research are required. Such studies should preferably be undertaken in a cross-cultural setting so that the findings can be compared between countries, thereby enabling discussions on how to optimize services in each country and between units with different levels of experience. Following the present project protocol, the results hopefully can be of significance when a comprehensive international model of care for rehabilitation of children and adolescents with SCI is developed.

\subsection{Aim}

The aim of this project protocol is twofold: 1) Plan and conduct a quantitative study to describe the content of inpatient pediatric SCI rehabilitation in ten rehabilitation units in seven countries; and 2) Plan and conduct a qualitative study representing all participating institutions that explores and describes psychosocial aspects of living with SCI in individuals aged 13-17 years.

\section{Methods}

\subsection{Design}

This is the protocol of a cross-sectional, descriptive project with a multiple method approach using one quantitative and one qualitative study to collect data 
in ten pediatric SCI rehabilitation units situated in Norway, Sweden, United States, Israel, PR China, Russia, and Palestine.

\subsection{Procedure and outcome measures}

Two contact persons at each unit will be assigned as local principal investigators (PIs), one for each part of the study. Inclusion criteria for PIs for both studies are the ability to speak English fluently as well as expert competence in neurological rehabilitation and/or experience working with pediatric SCI rehabilitation. Before data collection, all local contacts will be invited to a two-day workshop with the research group to review and refine data collecting instruments and methods.

To provide an overview of current practice of pediatric SCI rehabilitation in the participating units, the following characteristics will be described in a quantitative manner in study one: catchment area; funding; hospital setting; attachment to an acute SCI unit; provision of national, regional or state/provincial services; number of pediatric SCI rehabilitation beds; admittance and discharge procedures; availability of services; staff/patient ratio; content and intensity of rehabilitation programs; length of stay; measurement methods; follow-up services; health promotion; and pediatric SCI prevention acts. A web-based questionnaire developed by New et al. [25] will be further developed and extended to gather this information. The PIs at each unit will be responsible for collecting the data, using hospital administrators, rehabilitation staff, registries and other experts as sources.

For the qualitative data in study two, psychosocial aspects will be investigated through face-to-face interviews with adolescents 13-17 years of age. The interviews will be conducted in native languages by one of the local PIs who will also be responsible for collecting descriptive data from the participating adolescents (age, gender, age at injury, severity of injury, secondary complications, functional ability, use of aids and living situation).

A salutogenic approach with strength-based questions built on established principles and guidelines for psychosocial screening in young persons will be used to develop a semi-structured interview guide [39-42]. Appropriate open-ended questions will be included within main themes and key questions will be marked to ensure that they are asked by all interviewers. Interviewers will be instructed to focus on what is relevant for the young person and let the questions become part of a conversational interactive style.
A timeline of pictures illustrating the various themes in the interview guide will be used to facilitate the adolescent's reflections and experiences. The themes in the interview guide are acute care, primary rehabilitation, daily life, family, school, contact with friends, leisure time activities, peer learning, physical and psychological health, and plans for the future. The interviews will take place in the adolescent's home or another place chosen by the adolescent. Estimated time for the interviews is 60 minutes.

Pilot interviews will be conducted to ensure that all interviewers are familiar with the interview guide and the technical equipment, and that the interviews are conducted in a correct and efficient manner. All units will be given a digital voice recorder to tape the interviews and a transcription set to transcribe the taped interviews verbatim. The PIs will be responsible for translating the transcripts into English, either themselves or by another bilingual person with knowledge of SCI rehabilitation.

Data will be reported for both studies from the two PIs in each unit to the main PI (KSR) and research coordinator (SS) electronically. Ambiguities and clarification of responses will take place regularly using Skype, telephone, and email.

\subsection{Settings}

The project will be organized within the Sunnaas International Network in Rehabilitation (SIN) administration and the Department of Research, Sunnaas Rehabilitation Hospital, Norway. All participating units are collaborating within the well-established SIN network. The effectiveness of this collaboration within rehabilitation research has been demonstrated through a recent and similar multinational project on specialized stroke rehabilitation, the SIN Stroke project [36].

The participating rehabilitation units will provide descriptive data on their unit and the content of their specialized pediatric SCI rehabilitation. They will also conduct interviews with the adolescents who have been rehabilitated at their unit (Table 1).

\subsection{Participants in the qualitative study (study two)}

Adolescents with a diagnosis of SCI according to the International Standards for Neurological Classification of SCI with the ASIA Impairment Scale (AIS) [37], aged 13-17 years of age and irrespective of gender, will be invited to participate in the qualitative 
Table 1

The ten rehabilitation units which constitute Sunnaas International Network in Rehabilitation (SIN), who will all participate in the SIN Pediatric Spinal Cord Injury project (SINpedSCI)

\begin{tabular}{|c|c|c|c|}
\hline & Rehabilitation units & Country & City \\
\hline 1 & Sunnaas Rehabilitation Hospital & Norway & Oslo \\
\hline 2 & Rehab Station Stockholm & Sweden & Stockholm \\
\hline 3 & $\begin{array}{l}\text { H.R.H. Crown Princess Victoria's Children and Youth Hospital, } \\
\text { Linköping Hospital }\end{array}$ & Sweden & Linköping \\
\hline 4 & $\begin{array}{l}\text { Institute of Neuroscience and Physiology Rehabilitation Medicine, } \\
\text { Sahlgrenska University Hospital }\end{array}$ & Sweden & Gothenburg \\
\hline 5 & Rusk Rehabilitation, New York University Health Medical Center & USA & New York \\
\hline 6 & $\begin{array}{l}\text { Department of Pediatric Rehabilitation, The Edmond \& Lily Safra } \\
\text { Children's Hospital, The Chaim Sheba Medical Center }\end{array}$ & Israel & Tel-Hashomer \\
\hline 7 & $\begin{array}{l}\text { China Rehabilitation and Research Center (CRRC) and Capital } \\
\text { Medical University School of Rehabilitation Medicine }\end{array}$ & PR China & Beijing \\
\hline 8 & Bayi Rehabilitation Center & PR China & Chengdu \\
\hline 9 & Children's City Hospital & Russia & Petrozavodsk \\
\hline 10 & Bethlehem Arab Society for Rehabilitation and Surgery (BASR) & Palestine & Bethlehem \\
\hline
\end{tabular}

study. Inclusion criteria are adolescents with a traumatic or non-traumatic SCI sustained after the age of 7 years, discharged for at least 6 months from primary rehabilitation from one of the participating rehabilitation units, and willing to participate in the study. The adolescents have to be able to cooperate and communicate in their native language. Exclusion criteria are diagnosis of severe neurological conditions affecting the cognition of the adolescent, e.g., encephalocele or acquired brain injury.

\subsection{Sample size in the qualitative study (study two)}

A convenience sample consisting of at least 20 adolescents with SCI (approximately two from each unit) with experience related to the phenomena being studied will be chosen. The number of participants is guided by Kvale and Brinkmann [38] who consider $15 \pm 10$ participants a sufficient sample size to obtain information about the research phenomena and to yield a manageable amount of data in qualitative studies. Although generalization of qualitative findings is not a goal of qualitative research, qualitative researchers strive for transferability of their findings. To address this, a rich and detailed description of the adolescents' views will be provided.

\subsection{Ethical considerations}

Approval from the local ethical committees has been obtained from all the participating units and from the Regional Ethical Committee of Health in South-East Norway (2017/1867; 21 December 2017, 3 April 2019, 26 May 2020). Age appropriate infor- mation will be given, both in writing and verbally, to the adolescents and their parents, in accordance with the Helsinki Declaration [44]. Forms for written consent will be obtained from all participants and their parents in all clinics by the local PIs. The adolescents may withdraw from the study at any time.

\subsection{Data analysis}

The content of specialized rehabilitation (study one) will be described and compared with standards according to the SCIRE definition of specialized SCI rehabilitation [10], basic rules for the management of pediatric SCI [1], the ASIA guidelines for good pediatric SCI rehabilitation [17] and the WHO Standards for quality of care for children and young adolescents in health facilities $[15,16]$, in a qualitative manner. Descriptive analysis will be used on numerical data, with the median and interquartile range (IQR) reported for numerical data not normally distributed. The adolescents' qualitative data (study two) will be analyzed using thematic analysis, as developed by Braun and Clarke [43]. This is a method for identifying, analyzing, and reporting patterns within qualitative data.

\section{Discussion}

A multinational research project protocol with a focus on the content of pediatric SCI rehabilitation from the perspective of both the spinal rehabilitation unit and from the perspective of the young person with SCI is presented. 
The comparative design may contribute to knowledge about the content and quality of pediatric SCI rehabilitation with examples from ten rehabilitation units in seven countries and enhance discussions about how to optimize services in the different units and countries. The use of a quantitative approach is hopefully appropriate to gain valid and transferable knowledge on the organization and delivery of rehabilitation services, treatment systems, and outcome measures. The qualitative, explorative design targets how young people with SCI post rehabilitation perceive and experience the physical, psychosocial, and attitudinal circumstances and challenges that they meet in daily life and contributes to a deeper understanding of their experiences.

To the authors' knowledge, this study is the first comprehensive international study of services and systems of care from the perspective of spinal rehabilitation units as well as the perspective of young persons with sustained SCI. By exploring this topic from different perspectives and contexts, this project will contribute to a broader and more valid base of knowledge on pediatric SCI rehabilitation. Further, this project will enable comparison, discussion, and development of organizational models and quality of care and rehabilitation to safeguard and promote health, functioning, activity, participation, and quality of life. Data collected in different contexts also increases the transferability.

\section{Acknowledgments}

The study is funded by the Southern and Eastern Norway Regional Health Authority, Norwegian Ministry of Health and Care Services, Sunnaas Rehabilitation Hospital, and the participating units.

Special thanks to the following for valuable support: Einar Magnus Strand, CEO, Sunnaas Rehabilitation Hospital, Norway; Li JianJun, Head of China Rehabilitation Science Institute, former Head of China Rehabilitation Research Center, Beijing, PR China; Steven Flanagan, Chair, Rusk Rehabilitation, NYU Health Medical Center, NY, USA; Edmund Shehadeh, Head of Bethlehem Arab Society for Rehabilitation and Surgery, Palestine; Arkadi Rutgayzer, Head of Policlinika 2, Petrozavodsk, Russia; Amichai Brezner, Head of Department of Pediatric Rehabilitation, The Edmond \& Lily Safra Children's Hospital, Sheba Medical Center (SMC), Tel-Hashomer, Israel; Ofer Keren, Chaim Sheba Medical Center, Tel-Hashomer, Israel;
Per-Ola Rike, Sunnaas Rehabilitation Hospital, Norway.

\section{Ethical considerations}

Trial registration: Clinical Trials Gov, 12 June 2018: NCT04117854.

\section{Conflict of interest}

The authors have no conflicts of interest to report.

\section{References}

[1] Vogel LC, Zebracki K, Mulcahey MJ. Special considerations for rehabilitation of pediatric spinal cord injury. In: Chhabra HS, editor. ISCoS textbook in comprehensive management of spinal cord injuries. 1st ed. New Delhi: Wolters Kluwer; 2015.

[2] Parent S, Mac-Thiong JM, Roy-Beaudry M, Sosa JF, Labelle H. Spinal cord injury in the pediatric population: a systematic review of the literature. J Neurotrauma. 2011;28:1515-24. doi: 10.1089/neu.2009.1153

[3] Vogel CL, Zebracki K, Betz RR, Mulcahey MJ, editors. Spinal cord injury in the child and young adult. London: Wiley; 2015.

[4] New WP, Lee BB, Cripps R, Vogel LC, Scheinberg A, Waugh M-C. Global mapping for the epidemiology of paediatric spinal cord damage: towards a living data repository. Spinal Cord. 2019;57:183-97. doi: 10.1038/s41393-018-02 09-5

[5] Zebracki K, Vogel LC. Epidemiology of pediatric onset spinal cord injuries in the United States. In: RahimiMovaghar V, Jazayeri SB, Vaccaro AR, editors. Epidemiology of spinal cord injuries. New York: Nova Science Publishers; 2012;19-28.

[6] Vogel LC, Hickey K, Klaas S, Anderson C. Unique issues in pediatric spinal cord injury. Orthop Nurs. 2004;23:300-8. doi: 10.1097/00006416-200409000-00004

[7] NHS England [homepage on the Internet]. England: NHS standard contract for specialised rehabilitation for patients with highly complex needs (all ages); 2013 [updated 2013; cited 2020 May 23]. Available from: https://www.england. nhs.uk/wp-content/uploads/2014/04/d02-rehab-pat-highneeds-0414.pdf

[8] The Norwegian Institute of Public Health (NIPH) [homepage on the Internet]. Oslo, Norway: Health system review; 2014 [updated 2014 Sep 12; cited 2020 Mar 3]. Available from: https://www.fhi.no/en/pub1/2014/norway-health-sys tem-review2/.

[9] British Society of Rehabilitation Medicine (BSRM). Standards for rehabilitation services mapped on the national service framework for long-term conditions. London: British Society of Rehabilitation Medicine, Royal College of Physicians; 2009. Available from: https://www.bsrm.org.uk/ downloads/standardsmapping-final.pdf. 
[10] Spinal Cord Injury Rehabilitation Evidence (SCIRE) [homepage on the Internet]. Vancouver: Rehabilitation practices; 2010-2020 [cited 2020 May 23]. Available from: https://scireproject.com/evidence/rehabilitation-evidence/ rehabilitation-practices/

[11] Turner-Stokes L, Pick A, Nair A, Disler PB, Wade DT. Multi-disciplinary rehabilitation for acquired brain injury in adults of working age. Cochrane Database Syst Rev. 2015;(12);CD004170. doi: 10.1002/14651858.CD004 170.pub3

[12] Turner-Stokes L. Evidence for the effectiveness of multidisciplinary rehabilitation following acquired brain injury: a synthesis of two systematic approaches. J Rehabil Med. 2008;40:691-701. doi: 10.2340/16501977-0265

[13] The National Service Framework for Long-term Conditions. Department of Health; 2005. Available from: https:// www.gov.uk/government/publications/quality-standardsfor-supporting-people-with-long-term-conditions.

[14] British Society of Rehabilitation Medicine. Specialist neuro-rehabilitation services: providing for patients with complex rehabilitation needs. London: British Society of Rehabilitation Medicine, Specialised Neurorehabilitation Service Standards; 2010 [updated 2015 Apr 30]. Available from: https://www.bsrm.org.uk/downloads/specialised-neu rorehabilitation-service-standards-7-30-4-2015-forweb. pdf.

[15] WHO. Child and adolescent health. Children's rights in hospital - rapid-assessment checklist. Regional Office for Europe; 2015. Available from: https://www.euro.who.int/-data/assets/pdf_file/0004/342769/Check-list-Child-rightsin-hospital_layoutOPE.pdf.

[16] WHO. Standards for improving the quality of care for children and young adolescents in health facilities. Geneva: WHO Document Production Services; 2018. Available from: https://apps.who.int/iris/bitstream/handle/10665/272 346/9789241565554-eng.pdf?ua $=1$.

[17] American Spinal Injury Association (ASIA). Consumer guidelines. Available from: https://asia-spinalinjury.org/wp -content/uploads/2018/11/Consumer-Guidelines-v.-0308 2019.pdf.

[18] Schottler J, Vogel LC, Sturm P. Spinal cord injuries in young children: a review of children injured at 5 years of age and younger. Dev Med Child Neurol. 2012;54:1138-43. doi: 10.1111/j.1469-8749.2012.04411.x

[19] Mulcahey MJ, Gaughan JP, Betz RR, Samdani AF, Barakat N, Hunter LN. Neuromuscular scoliosis in children with spinal cord injury. Top Spinal Cord Inj Rehabil. 2013;19:96103. doi: 10.1310/sci1902-96

[20] Geuther M, Grassnes L, Mach O, Klein B, Högel F, Voth $\mathrm{M}$, et al. Functional outcome after traumatic cervical spinal cord injury is superior in adolescents compared to adults. Eur J Paediatr Neurol. 2019;23:248-53. doi: 10.1016/j.ejpn.2018.12.001

[21] Fawcett JW, Curt A, Steeves JD, Coleman WP, Tuszynski $\mathrm{MH}$, Lammertse D, et al. Guidelines for the conduct of clinical trials for spinal cord injury as developed by the ICCP panel: spontaneous recovery after spinal cord injury and statistical power needed for therapeutic clinical trials. Spinal Cord. 2007;45:190-205. doi: 10.1038/sj.sc.3102007

[22] Augutis M, Levi R, Asplund K, Berg-Kelly K. Psychosocial aspects of traumatic spinal cord injury with onset during adolescence: a qualitative study. J Spinal Cord Med. 2007;30(suppl 1):55-64. doi: 10.1080/10790268.2007.1175 3970
[23] Neinstein LS, Katzman D, editors. Adolescent and young adult health care: a practical guide. 6th ed. Philadelphia: Wolters Kluwer; 2016.

[24] Augutis M, Levi R. Pediatric spinal cord injury in Sweden; incidence, etiology and outcome, 2003. Spinal Cord. 2003;41:328-36. doi:10.1038/sj.sc.3101478

[25] New PW, Townson A, Scivoletto G, Post MWM, EriksHoogland I, Gupta A, et al. International comparison of the organization of rehabilitation services and systems of care for patients with spinal cord injury. Spinal Cord. 2013;51:33-9. doi: 10.1038/sc.2012.82

[26] Vandenbroucke JP, von Elmet E, Altman D, Götzsche P, Mulrow C, Pocock SJ, et al. Strengthening the Reporting of Observational studies in Epidemiology (STROBE): explanation and elaboration. PLoS Med. 2007;4:e297. doi: 10.1371/journal.pmed.0040297

[27] New PW. The assessment and selection of potential rehabilitation patients in acute hospitals: a literature review and commentary. Open Rehab J. 2009;2:24-34. doi: 10.2174/18 74943700902010024

[28] Slade A, Tennant AA, Chamberlain MA. A randomised controlled trial to determine the effect of intensity of therapy upon length of stay in a neurological rehabilitation setting. J Rehabil Med. 2002;34:260-66. doi: 10.1080/165019702760390347

[29] Augutis M, Abel R, Levi R. Pediatric spinal cord injury in a subset of European countries. Spinal Cord. 2006;44:106-12. doi: $10.1038 /$ sj.sc.3101793

[30] World Health Organization and International Spinal Cord Society. Bickenbach CBJ, Brown D, Burns A, Campbell $\mathrm{R}$, Cardenas D, Charlifue S, et al., editors. International perspectives on spinal cord injury. Geneva: WHO Press; 2013. Available from: https://www.who.int/disabilities/ policies/spinal_cord_injury/en/.

[31] Biering-Sørensen F, Charlifue S, DeVivo M, Noonan $\mathrm{V}$, Post M, Stripling $\mathrm{T}$, et al. International Spinal Cord Injury Data Sets. Spinal Cord. 2006;44:530-4. doi: 10.1038/sj.sc.3101958

[32] Kirshblum SC, Burns SP, Biering-Sorensen F, Donovan $\mathrm{W}$, Graves DE, Jha A, et al. International standards for neurological classification of spinal cord injury (revised 2011). J Spinal Cord Med. 2011;34:535-46. doi: $10.1179 / 204577211 X 13207446293695$

[33] Carroll A, Vogel LC, Zebracki K, Noonan VK, BieringSørensen F, Mulcahey MJ. Relevance of the international spinal cord injury basic data sets to youth: an interprofessional review with recommendations. Spinal Cord. 2017;55:875-81. doi: 10.1038/sc.2017.14

[34] Hwang M, Augutis M, Sadowsky C, Höfers W, Vogel LC, Post M, et al. The International Spinal Cord Injury Pediatric Activity and Participation Basic Data Set. Spinal Cord Ser Cases. 2019;5:91. doi: 10.1038/s41394-019-0230-8

[35] Carney J, Fisher R, Augutis M, Charlifue S, BieringSørensen F, Höfers W, et al. Development of the International Spinal Cord Injury/Education Basic Data Set. Spinal Cord Ser Cases. 2019;5:87. doi: 10.1038/s41394019-0229-1

[36] Langhammer B, Becker F, Zhang T, Stibrant-Sunnerhagen $\mathrm{K}$, Tong Z, Du X, et al. Specialized stroke rehabilitation services in seven countries. Preliminary results from nine rehabilitation centers. Int J Stroke. 2015;10:1236-46. doi: 10.1111/ijs.12599

[37] Kirshblum S, Burns SP, Biering-Sorensen F, Donovan W, Graves DE, Jha A, et al. International standards 
for neurological classification of spinal cord injury (revised 2011). J Spinal Cord Med. 2011;34:535-44. doi: 10.1179/204577211X13207446293695

[38] Kvale S, Brinkman S. Interviews - learning the craft of qualitative research interviewing. 2nd ed. Los Angeles, CA: Sage; 2009.

[39] EuTEACH [homepage on the Internet]. Lausanne: European training in effective adolescent care and health [updated 2019 Sep 19; cited 2020 May 23]. Available from: https://www.unil.ch/euteach/en/home.html.

[40] HEEADSSS assessment [homepage on the Internet]. Auckland: Goodfellow unit [updated 2017; cited 2020 May 23]. Available from: http://www.goodfellowunit.org/coursepage/introduction-heeadsss-assessment/assessment.

[41] American Academy of Pediatrics. HEADSSS assessment: risk and protective factors. Available from: http://www.heardalliance.org/wp-content/uploads/2011/ 04/HEADSS.pdf.
[42] Clinical Practice Guidelines. Royal Children's Hospital Melbourne [homepage on the Internet]. Melbourne: Engaging with and assessing the adolescents' patient [updated 2019 Sep 19; cited 2020 May 23]. Available from: https://www.rch.org.au/clinicalguide/guideline_index/ Engaging_with_and_assessing_the_adolescent_patient/

[43] Braun V, Clarke V. Using thematic analysis in psychology. Qual Res Psychol. 2006;3:77-101. doi: 10.1191/ 1478088706qp063oa

[44] World Medical Association (WMA) [homepage on the Internet]. WMA Declaration of Helsinki - Ethical principles for medical research involving human subjects [updated $2018 \mathrm{Jul}$ 9; cited 2020 May 23]. Available from: https://www.wma.net/policies-post/wma-declarationof-helsinki-ethical-principles-for-medical-researchinvolving-human-subjects/. 